\title{
Induction of high-mobility group Box-1 in vitro and in vivo by respiratory syncytial virus
}

\author{
Sara Manti ${ }^{1}$, Terri J. Harford ${ }^{1}$, Carmelo Salpietro ${ }^{2}$, Fariba Rezaee ${ }^{1}$ and Giovanni Piedimonte ${ }^{1}$
}

BACKGROUND: Despite decades that have passed since its discovery, accurate biomarkers of respiratory syncytial virus (RSV) disease activity and effective therapeutic strategies are still lacking. The high-mobility group box type 1 (HMGB1) protein has been proposed as a possible link between RSV and immune system, but only limited information is currently available to support this hypothesis.

METHODS: Expression of HMGB1 gene and protein was analyzed by quantitative PCR, enzyme-linked immunosorbent assay (ELISA), western blot, immunocytochemistry, and confocal microscopy in immortalized and primary human bronchial epithelial cells, as well as in rat pup lungs. The role of HMGB1 in RSV infection was explored using glycyrrhizin, a selective HMGB1 inhibitor.

RESULTS: RSV infection strongly induced HMGB1 expression both in vitro and in vivo. Glycyrrhizin dose-dependently inhibited HMGB1 upregulation in both RSV-infected immortalized and primary human bronchial epithelial cells, and this effect was associated with significant reduction of viral replication.

CONCLUSION: Our data suggest that HMGB1 expression increases during RSV replication. This seems to have a critical pathogenic role as its selective inhibition virtually modified the infection. These observations provide further insight into the pathophysiology of RSV infection and uncover a potential biomarker and therapeutic target for the most common respiratory infection of infancy.

$\mathbf{R}$ espiratory syncytial virus (RSV), an enveloped, nonsegmented, negative-sense RNA virus of the Paramyxoviridae family, is the most common respiratory pathogen in infants and young children worldwide (1). Prospective epidemiologic studies have suggested a strong association between RSV lower respiratory tract infection during infancy and subsequent development of recurrent wheezing and asthma in childhood (2). Recent research in animal models has shown vertical transmission of RSV from the mother's respiratory tract to the fetal lungs, with postnatal persistence of the virus linked to persistent airway hyper-reactivity (3).
Despite many years of research, we still lack reliable biomarkers of disease activity, as well as effective vaccines and therapeutic strategies.

Recently, the high-mobility group box type 1 (HMGB1) protein has been proposed as a biomarker potentially able to elucidate the link between RSV and chronic airway dysfunction $(4,5)$. HMGB1 is an inflammation marker of the alarmins family promoting immediate immune response to tissue damage (6), and is one of the most important damageassociated molecular pattern molecules, initiating and perpetuating immune responses in infectious and noninfectious inflammatory diseases (7). Its role is to act as a "danger signal" orchestrating homeostatic defensive responses in damaged tissues (6).

The major structural features of HMGB1, a 30-kDa nuclear and cytosolic ubiquitous protein, are its two DNA-binding domains, termed $\mathrm{A}$ and $\mathrm{B}$ box, and a negatively charged C-terminal acidic region. HMGB1 contains two nuclear localization sequences, resides in the nucleus, and functions as a nonhistone chromatin-binding protein (8). Early work demonstrated that HMGB1 stabilizes chromatin structure and modulates gene transcription by bending the DNA helical structure (9). However, HMGB1 can also be localized to the cytosolic compartment, implicating that it might also have important functions outside the nucleus (7).

As a consequence of infection or apoptosis, HMGB1 is released in the extracellular compartment either by passive release from necrotic cells or active production by macrophages, dendritic cells, and natural killer cells (10). By binding to toll-like receptors (TLR) 2 and 4, and the receptor for advanced glycation end products (RAGE) (11), HMGB1 upregulates the synthesis of inflammatory cytokines, elicits chemotaxis of inflammatory cells, and supports proliferation, chemotaxis, and synthesis of metalloproteinases by stromal fibroblasts (12), thereby contributing to the pathogenesis of both acute and chronic diseases (13).

Although it has been reported that HMGB1 is critically involved in multiple stages of several DNA (herpes simplex virus type 2) and RNA (West Nile virus, dengue) viral infections, limited data are available on its role during

\footnotetext{
${ }^{1}$ Center for Pediatric Research, Pediatric Institute and Children's Hospital, Cleveland Clinic Foundation, Cleveland, Ohio; ${ }^{2}$ Department of Human Pathology of Adult and Developmental Age “Gaetano Barresi”, Unit of Paediatric Genetics and Immunology, University Hospital of Messina, Messina, Sicily, Italy. Correspondence: Fariba Rezaee (rezaeef@ccf.org)

Received 6 April 2017; accepted 31 December 2017; advance online publication 21 February 2018. doi:10.1038/pr.2018.6
} 
RSV infection (4,5). Hou et al. reported increased HMGB1 levels in the lung tissue of RSV-infected mice (5). In addition, HMGB1 in infants with RSV bronchiolitis tends to reach higher concentrations compared with other viral infections (14). Thus, we hypothesized that HMGB1 is essential for the establishment of productive RSV infection, and, to this end, we studied its gene and protein expression in human bronchial epithelial cells infected in vitro and in the lungs of rat pups infected in the neonatal period. Furthermore, we selectively inhibited HMGB1 activity in RSV-infected cells using glycyrrhizin and studied its effect on viral replication.

\section{METHODS}

\section{Airway Epithelial Cell Culture}

16HBE140-, SV-40 virus-transformed immortalized human bronchial epithelial (called thereafter 16HBE) cells were seeded on collagen-coated Transwell inserts (Costar, Corning, NY) or on 12-well cell culture plates, and were cultured in D-MEM high glucose containing $10 \%$ heat-inactivated fetal calf serum, penicillin $(100 \mathrm{U} / \mathrm{ml})$, streptomycin $(100 \mu \mathrm{g} / \mathrm{ml})$, and HEPES $(0.015 \mathrm{~mol} / \mathrm{l})$ at $37^{\circ} \mathrm{C}$ in humidified $5 \% \mathrm{CO}_{2}$ atmosphere $(15,16)$. Primary normal human bronchial epithelial (NHBE) cells isolated from lungs of de-identified deceased donors, provided by the International Institute for the Advancement of Medicine (IIAM), were grown in defined media and utilized between passages 3 and 6 (ref. 15).

\section{Viral Infection of Epithelial Cell Cultures}

Recombinant RSV- $\mathrm{A}_{2}$ expressing the red fluorescent protein (RFP) gene (rrRSV) was kindly provided by Mark Peeples (Nationwide Children's Hospital, Columbus, $\mathrm{OH}$ ) and Peter Collins (National Institutes of Health, Bethesda, MD) $(17,18)$. Expression of viable RFP requires successful full-length RSV replication, and the rrRSV strain construct (BN1) used in these experiments is described elsewhere (19). Stock rrRSV was propagated using HEp-2 cells (ATCC CCL-23; American Type Culture Collection, Manassas, VA) in $1 \times$ DMEM with $10 \%$ fetal bovine serum. HEp- 2 cells at $70 \%$ confluence were inoculated, harvested, and titrated as described previously (3). To obtain virus-free inoculum, HEp-2 cells were identically cultured and harvested. In addition, cells were treated with glycyrrhizin, ammonium salt $5 \mathrm{~g}$, EMB Millipore, Billerica, MA. Glycyrrhizin at $50 / 100 \mu \mathrm{M}$ was applied to $16 \mathrm{hbec}$ and human primary cells, both simultaneously and successively infected with virus.

\section{Animals}

Ten-week-old, pathogen-free Fisher 344 (F-344) rats housed under barrier conditions in a BSL-2 facility were used. Rats were housed in polycarbonate isolation cages on racks providing positive individual ventilation with class-100 air at the rate of one cage change per minute (Lab Products, Seaford, DE). All manipulations were conducted inside class-100 laminar flow hoods. Ten-day-old pups were inoculated with $4.0 \times 10^{5}$ plaque-forming unit of rrRSV or an equal volume of sterile inoculum by intratracheal instillation, as previously described (3). Rats were killed 5 days after infection and the lungs were removed for analysis. All experimental protocols and procedures utilized in this study were reviewed and approved before implementation by the Cleveland Clinic Institutional Animal Care and Use Committee, and adhered to the NIH Guide for the Care and Use of Laboratory Animals.

\section{Extraction of RNA and Quantitative Real-Time Analysis}

Total RNA was isolated from epithelial cells using RNeasy Kit (Qiagen, Germantown, MD, USA) according to the manufacturer's instructions. RNA was reverse-transcribed into cDNA using the High Capacity cDNA Reverse Transcription Kit (Applied Biosystems, Foster City, CA) and cDNA was used to carry out qPCR reaction using the CFX connect Real Time PCR System (Bio-Rad,
Hercules, CA). All experiments were carried out in quadruplicate. Relative expression levels of mRNA were calculated with the $2-\Delta \Delta \mathrm{Ct}$ method and were normalized to actin.

The primer pairs were designed on the basis of previously published protocols to discriminate CDNA-generated PCR products from genomic DNA contamination (20). Random primers were used for the reverse transcription (RT) reaction. RSV primers (F: 5'GCGATGTCTAGGTTAGGA-3' and R: 5'-GCTATGTCCTTGGG TAGT- $3^{\prime}$ ) target the sequence $1,303-1,712 \mathrm{bp}$ of the RSV serotype A2 genome (GenBank accession number M11486), which encodes for the viral nucleocapsid (N) protein. Because the GenBank sequence is missing 34 nucleotides in the $3^{\prime}$ leader region, the position of the target sequence on the complete RSV-A2 genome is $1,347-1,756$. The $\mathrm{N}$ protein is a structural protein located inside the virion tightly bound to the RNA strand and is typically targeted to detect the presence of the whole virus in infected cells.

Primers for human HMGB1 (forward: 5'-TCGGCCTTCT TCCTCTTCT-3'; reverse 5'-CCACATCTCTCC CAGTTTCTTC $-3^{\prime}$ ); and rat HMGB1 (forward: 5'-AGTTTCCTGAGCAATCCG TAT-3'; reverse: 5'-TGTATCCCCAAAAGCGTGAG-3') were designed and synthesized by Integrated DNA Technologies (Coralville, IA).

\section{Protein Electrophoresis and Immunoblotting}

Proteins were separated on SDS-PAGE gel (Bio-Rad), transferred onto polyvinylidene difluoride membranes, and blocked in Trisbuffered saline containing $0.1 \%$ Tween- 20 with $5 \%$ nonfat dry milk. Membranes were incubated overnight at $4{ }^{\circ} \mathrm{C}$ with anti-HMGB-1 (Santa Cruz Biotechnologies, Dallas, TX), followed by anti-rabbit secondary antibody conjugated with horseradish peroxidase $(1: 2,000)$. Blots were developed with an enhanced chemiluminescence reagent kit (Pierce West Pico; Thermo Scientific, Waltham, MA) according to the manufacturer's instructions.

\section{Immunofluorescence Staining}

Epithelial cells were fixed in $4 \%$ paraformaldehyde (PFA), permeabilized, and probed with anti-HMGB1 antibody, followed by staining with a secondary antibody conjugated with Alexa- 488 . Nuclei were stained with 4',6-Diamidine-2'-phenylindole dihydrochloride (DAPI). Images were acquired using upright fluorescent or confocal microscope (Leica Microsystems, Wetzlar, Germany) with a 405-diode laser to excite DAPI and a HeNe laser to excite the secondary antibody. Cells were visualized using a $\times 40$ or $\times 63 / 1.4$ oil objective. Densitometry and plot profiles were acquired using the NIH ImageJ software (Bethesda, MD).

\section{Enzyme-Linked Immunosorbent Assay}

Supernatant, cell lysates, and rat lung concentrations of HMGB1 were measured with a commercially available enzyme-linked immunosorbent assay (ELISA) kit (LifeSpan BioSciences, Seattle, WA) according to the manufacturer's instructions. Detection limit for $\mathrm{HMGB} 1$ was $0.16-10 \mathrm{ng} / \mathrm{ml}$.

\section{Statistical Analysis}

All experiments were repeated three times. Data are representative of three or more experiments and are presented as means \pm SEMs. Significance was considered at a $P$ value of less than 0.05 . Comparisons between two groups were performed with unpaired Student's $t$-test. Multiple comparisons were performed by ANOVA followed by post hoc analysis with the Dunnett's test using the software GraphPad Prism version 5.0 (La Jolla, CA). The densitometry analysis was performed using ImageJ and statistical analysis was performed using Graphpad Prism Version 5 using oneway ANOVA analysis Tukey's multiple comparison test. $P$ values less than 0.05 were considered statistically significant. 



Figure 1. HMGB1 expression in rrRSV-infected 16HBE cells. HMGB1 mRNA transcripts and protein expression were measured by qPCR and western blot analysis in human bronchial epithelial cells infected with rrRSV at MOI of 1 for $48 \mathrm{~h}$. (a) HMGB1 gene expression was upregulated at $3 \mathrm{~h}$ post infection, followed by reduced expression at 6,24 , and $48 \mathrm{~h}$. $\beta$-actin gene was used as the housekeeping control for transcript normalization. Data are expressed as mean \pm SEM. ${ }^{* *} P<0.001$ compared with non-infected cells. (b) HMGB1 protein expression was upregulated at $6 \mathrm{~h}$ post infection, before returning to baseline at 24 and $48 \mathrm{~h}$ post infection. (c) Densitometric analysis. Data are expressed as mean \pm SEM. ${ }^{*} P<0.05,{ }^{* *} P<0.01$ compared with time point $0 \mathrm{~h}$. All experiments were repeated at least three times. HMGB1, high-mobility group box type 1; qPCR, quantitative PCR; $\mathrm{MOI}$, multiplicities of infection; rrRSV, red fluorescent protein (RFP) gene.

\section{RESULTS}

In vitro: $16 \mathrm{HBE}$ cells infected with rrRSV at multiplicity of infection (MOI) of 1 showed significant upregulation of HMGB1 gene expression at $3 \mathrm{~h}$ post infection $(P<0.001)$, with return to baseline by $6 \mathrm{~h}$ (Figure 1a). Accordingly, western blot analysis confirmed increased HMGB1 protein in rrRSV-infected 16HBE, peaking at $6 \mathrm{~h}(P<0.001)$ and waning by $24 \mathrm{~h}$ (Figure $1 \mathrm{~b}, \mathrm{c}$ ). Immunofluorescence of $16 \mathrm{HBE}$ cells infected with RSV at MOI of 1 showed localization of HMGB1 into the nuclei with a peak at $6 \mathrm{~h}$ (Figure 2a). Densitometry analysis of HMGB1 expression revealed increased overall expression at $6 \mathrm{~h}$ before a decline at $24 \mathrm{~h}$ (Figure 2b). 16HBE has been frequently used as a model system of the airways for different cell signaling studies. To compare the results obtained with 16HBE, we also used differentiated primary NHBE cells. Similar to immortalized cells, immunofluorescence staining and confocal microscopy of primary NHBE cells infected with RSV at MOI of 1 resulted in an increase in HMGB1 expression in both nuclear and cytosolic regions in shorter time points with further localization of majority of HMGB1 into the cytosolic location at $24 \mathrm{~h}$ post infection.

In vivo: $\mathrm{qPCR}$ analysis of HMGB1 gene expression in rat lung homogenates measured a doubling of mRNA transcripts 5 day post inoculation of rrRSV, compared with age-matched mock-infected controls dosed with virus-free medium
$(P<0.01$; Figure $3 a)$. ELISA showed significantly higher HMGB1 protein concentrations in the lungs of rrRSVinfected pups $(P<0.01$; Figure $3 \mathbf{b})$. Western blot analysis confirmed that, although HMGB1 protein was weakly detected in pathogen-free lungs, its concentration was threefold higher in rrRSV-infected lungs $(P<0.001$; Figure $3 c, d)$.

In vitro: to determine whether HMGB1 has a causal role during rrRSV infection, we used the specific inhibitor glycyrrhizin to block HMGB1 activity in 16HBE cells and NHBE cells infected simultaneously with rrRSV. Glycyrrhizin decreased the number of RFP-positive cells in both 16HBE cells (Figure $4 \mathbf{a}, \mathbf{b}$ ) and NHB cells (Figure $5 \mathbf{a}, \mathbf{b}$ ) in a dosedependent manner. This profound effect of HMGB1 on rrRSV-infected cells was confirmed in NHBE cells by qPCR $(P<0.001$; Figure 5c). Similarly, western blotting confirmed that glycyrrhizin prevents rRSV-induced HMGB1 protein upregulation (Figure 5d).

\section{DISCUSSION}

This study shows that HMGB1 expression increases both in vitro and in vivo as a function of RSV infection and its localization reflects different phases of the replicative cycle, which may allow the use of local or systemic levels as a biomarker of disease activity. More importantly, this is the first study indicating that HMGB1 synthesis is an essential 
a Time post infection (h)
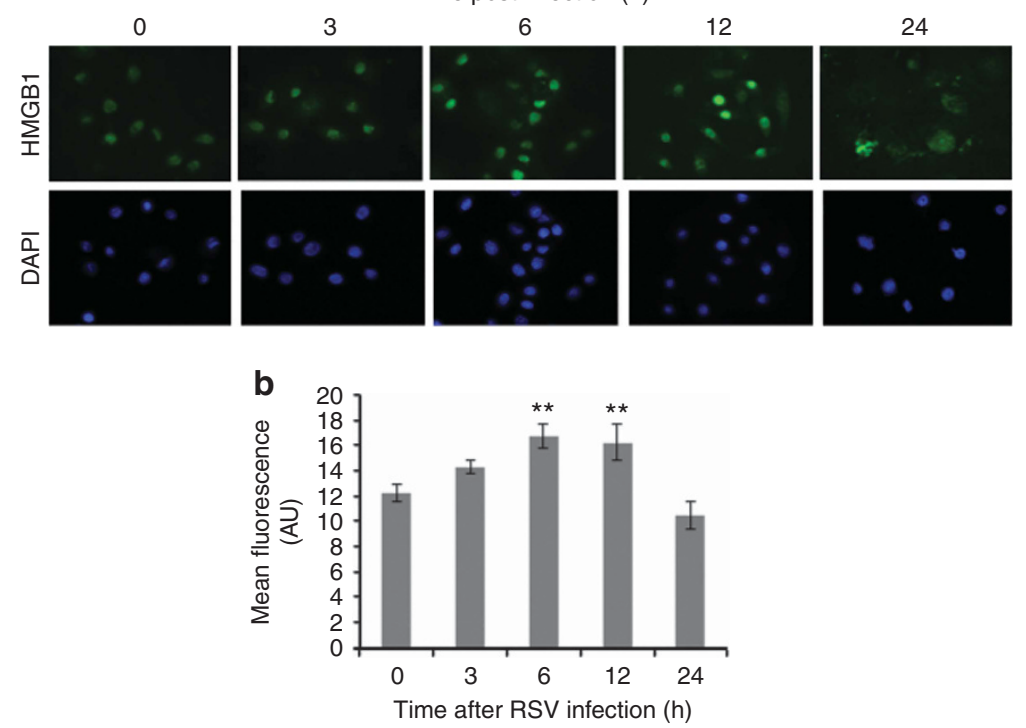

C

Time post infection (h)
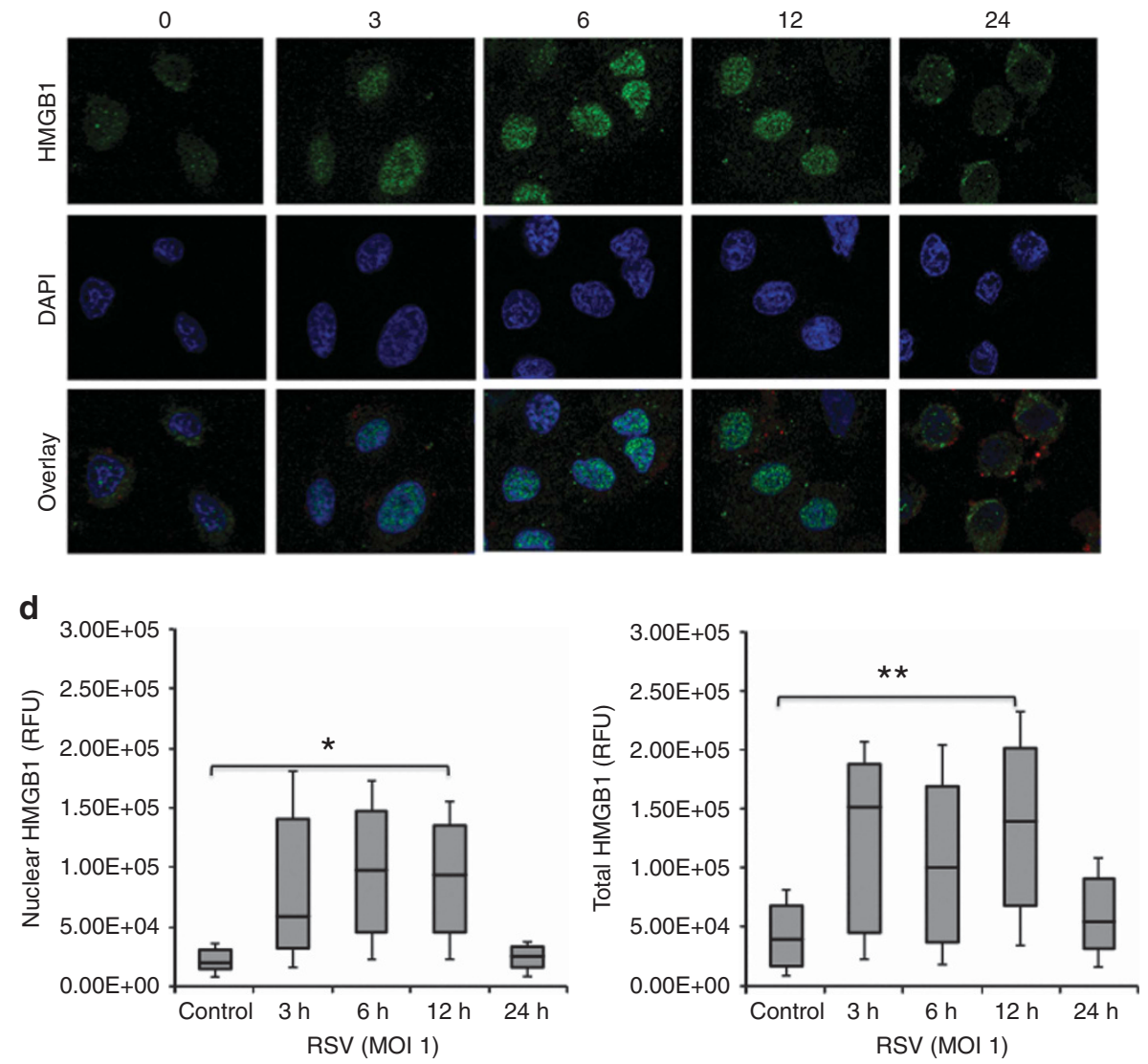

step of RSV replication. Consequently, selective inhibition of this protein in infected human bronchial epithelium drastically reduces the number of RSV-infected cells, thereby providing a novel therapeutic target for this common infection. Through the activity of the G and F glycoproteins, RSV attaches to and enters host cells, and 4-6 h after infection begins transcription and replication of viral genome, reaching a peak $\sim 20 \mathrm{~h}$ after infection. Synthesis and release of viral cytoplasmic particles containing RSV RNA and proteins start $12 \mathrm{~h}$ after viral entry and persist up to $48 \mathrm{~h}$ after infection.

In airway epithelial and immune cells, RSV is detected by three types of pattern recognition receptors, including retinoic acid-inducible gene-I-like receptors, nucleotide-binding oligomerization domain-like receptors, and TLRs, all promoting 
Figure 2. HMGB1 localization in rrRSV-infected airway epithelial cells. Human 16HBE and primary bronchial epithelial cells were infected with rrRSV at an MOI of 1 or control medium at the indicated time points. (a) HMGB1 in 16HBE cells was visualized in cells $24 \mathrm{~h}$ post infection by immunofluorescence showing localization of HMGB1 into the nuclei with a peak at $6 \mathrm{~h}$ (b) as evidenced by densitometry; ${ }^{* *} P<0.01$ compared with time zero. (c) Primary HBE cells by confocal microscopy showed localization of HMGB1 expression into the nuclei at 3 and $6 \mathrm{~h}$, in the cytoplasm starting at $12 \mathrm{~h}$, and on plasma membranes at $24 \mathrm{~h}$. (d) HMGB1 nuclear translocation was performed by densitometric analysis, reporting the percentage overlap between GFP and DAPI signals, reflecting the nuclear and total HMGB1 translocation at 3, 6, 12, and $24 \mathrm{~h}$ post infection; ${ }^{*} P<0.05$, ${ }^{* *} P<0.01$. All experiments were repeated at least three times. GFP, green fluorescent protein; HMGB1, high-mobility group box type $1 ;$ MOI, multiplicity of infection; rrRSV, red fluorescent protein (RFP) gene.
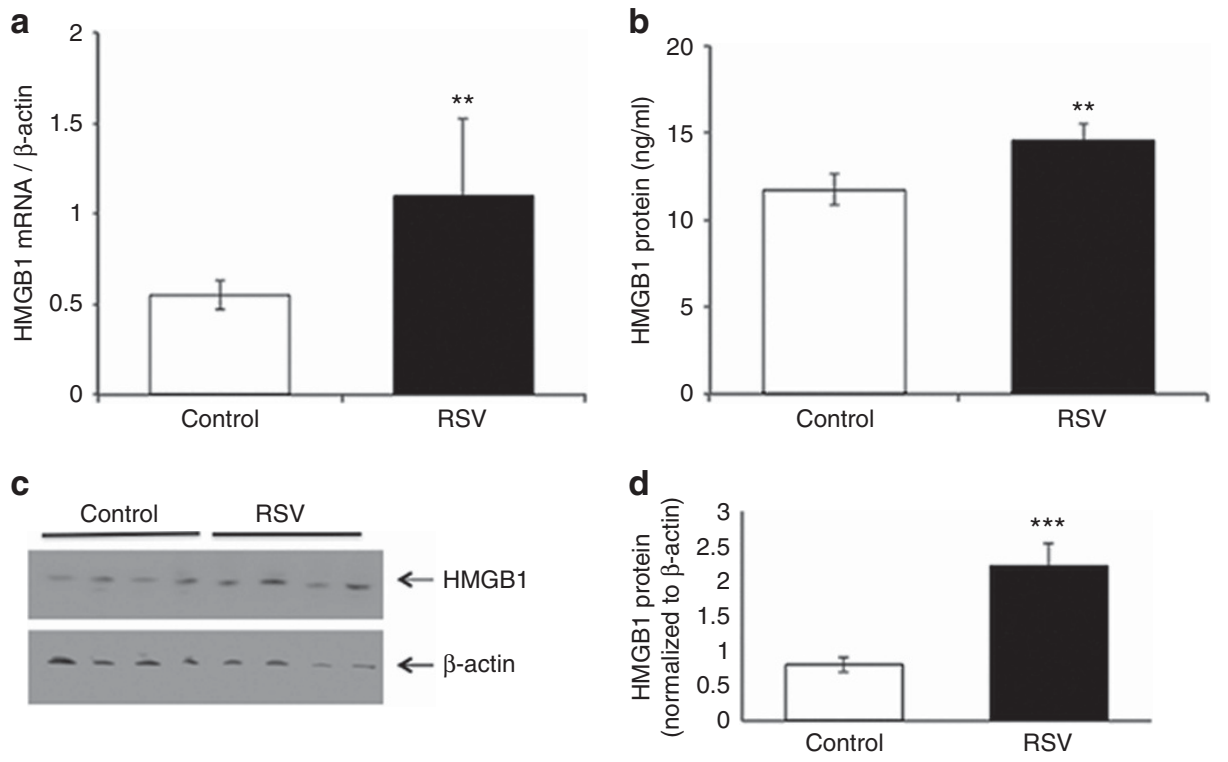

Figure 3. HMGB1 expression in rat lungs. (a) qPCR and (b) ELISA analyses were performed to determine, respectively, mRNA and protein expression of HMGB1 in lung tissues of rat pups infected with rrRSV on 10 days of life and killed 5 days later. Both HMGB1 mRNA and protein increased significantly in the lungs of rrRSV-infected rats compared with pathogen-free controls. (c) Increased HMGB1 protein expression in rrRSV-infected rat lungs was confirmed by western blot. (d) Densitometry analysis. Data are expressed as mean \pm SEM. ${ }^{* *} P<0.01,{ }^{* *} P<0.001$ compared with noninfected cells. All experiments were repeated at least three times. ELISA, enzyme-linked immunosorbent assay; HMGB1, high-mobility group box type 1; qPCR, quantitative PCR; rrRSV, red fluorescent protein (RFP) gene.
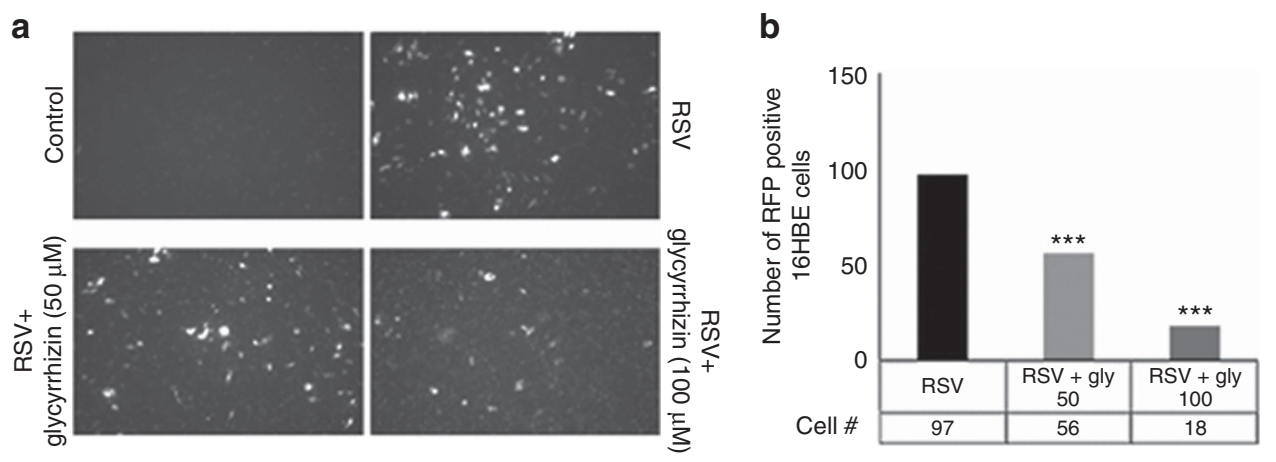

Figure 4. Effect of glycyrrhizin on rrRSV infection in 16HBE cells. (a) Representative pictures of rrRSV-infected 16HBE and non-infected controls treated with the HMGB1 antagonist glycyrrhizin $(50$ or $100 \mu \mathrm{M})$ or vehicle. The density of cells exhibiting red fluorescence produced by active viral replication was significantly reduced by glycyrrhizin in a dose-dependent manner. (b) Bar graph showing the number of rrRSV-positive cell in each group. Data are expressed as mean \pm SEM. ${ }^{* * *} P<0.001$ compared with RSV group. All experiments were repeated at least three times. HMGB1, highmobility group box type 1; rrRSV, red fluorescent protein (RFP) gene.

and sustaining nonspecific and/or specific immune response against RSV (21). TLRs induce immediate release of proinflammatory cytokines and inflammasome components that, in turn, amplify the immune response via late synthesis of alarmins, such as HMGB1 (ref. 22). The different timing of conventional cytokines and HMGB1 expression explains how 


\section{Articles | Manti et al.}

a
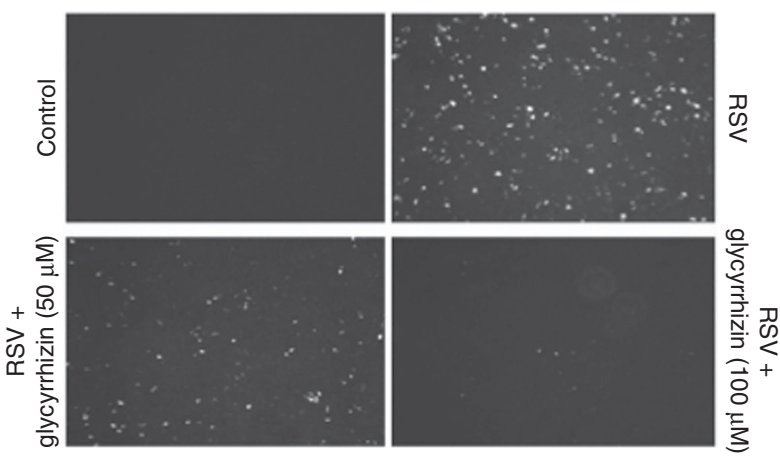

C

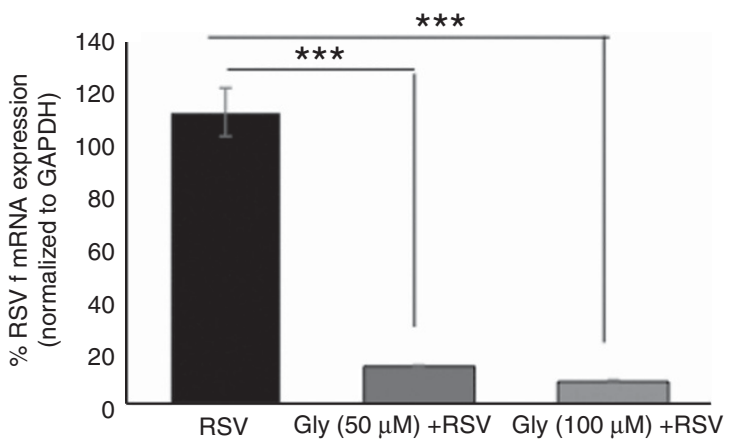

b

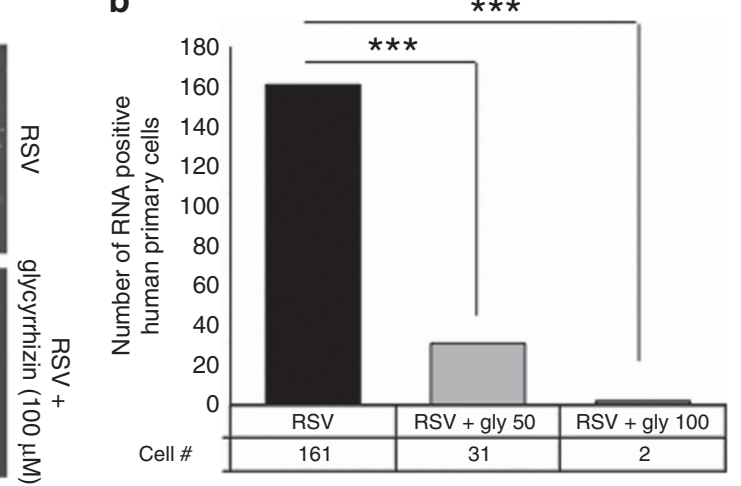

d

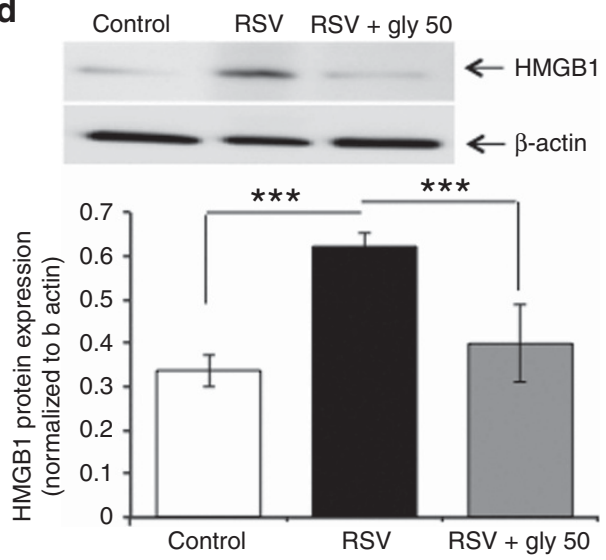

Figure 5. Effect of glycyrrhizin on rrRSV infection in human primary cells. (a) Fluorescent microscopy of human primary cells treated with the HMGB1 antagonist glycyrrhizin $(50$ or $100 \mu \mathrm{M})$. (b) The number of cells exhibiting the red fluorescence produced by active viral replication was significantly reduced by glycyrrhizin in a dose-dependent manner. (c) Consistently, qPCR measured a significant reduction in RSV F RNA levels in response to treatment with glycyrrhizin. (d) Western blot analysis with corresponding densitometry demonstrated downregulation of HMGB1 protein expression upon glycyrrhizin treatment. Data are expressed as mean \pm SEM. ${ }^{* * *} P<0.001$ compared with RSV group. All experiments were repeated at least three times. HMGB1, high-mobility group box type 1; qPCR, quantitative PCR; rrRSV, red fluorescent protein (RFP) gene; RSV, respiratory syncytial virus.

the former mediate acute inflammation, whereas the latter is not only involved in the early inflammatory phase but also maintains chronic inflammatory response (23). In particular, a previous study reported that HMGB1 expression in vivo starts within $3-8 \mathrm{~h}$ after inflammatory and/or infectious stimuli, and then increases progressively from 16 to $32 \mathrm{~h}$ (ref. 24). In accordance with these findings, we noted in vitro that: (i) HMGB1 mRNA levels sharply increased at $3 \mathrm{~h}$, during viral entry into living cells; (ii) nuclear HMGB1 localization peaked approximately at $6 \mathrm{~h}$, in parallel with viral transcription and replication; and (iii) $24 \mathrm{~h}$ after infection, during the release of viral cytoplasmic particles, HMGB1 migrated from the nucleus to the cytosol and plasma membranes.

Depending on its localization, HMGB1 exerts different time-dependent effects during viral infections. When expressed in the nucleus, HMGB1 acts as a nuclear enhancer for transcription factors and viral rolling circle replication as seen during adenovirus (25) and parvovirus (26) infections. When in the cytoplasm, HMGB1 is detrimental to the host response, reducing resistance to infections like influenza
A (27) and H1N1 (ref. 28). Moving toward the plasma membrane, HMGB1 binds RAGE, a receptor also expressed in epithelial cell cultures (29) and able to exacerbate RSV disease by amplifying the expression of proinflammatory agents. RAGE deficiency has been associated with viral-induced asthma phenotype in a mouse model (30). Our data also confirmed nuclear HMGB1 localization during the early phase of infection, which has been shown to be a critical initial event for an efficient viral cycle (4).

Supporting a pathogenic role of HMGB1 in RSV infection, we found increased HMGB1 expression in lung tissues of rat pups infected with RSV. It has been previously argued that an exogenous or endogenous immunogenic stimulus will activate the innate immune system only if able to induce the release of alarmins, like HMGB1 (ref. 31). By binding to its cognate receptors, HMGB1 modifies cell functions not only with direct autocrine/paracrine activity (32), but also through indirect potentiation of other inflammatory pathways, e.g., activation of multiple pattern-recognition receptors like TLR2 and TLR4 (ref. 32); release of proinflammatory cytokines like 


\section{HMGB1 signaling in RSV infection}

IL-1, IL-6, and IL-8 (ref. 33); and interference with T-cell responses inducing $\mathrm{TH} 1$ polarization (34).

Furthermore, Lei et al. (35) reported that increased HMGB1 levels are associated with overexpression of NGF, the prototypical neurotrophic factor known to be responsible for the development of neurogenic inflammation and airway hyper-reactivity during and after early-life infection by RSV (36). Therefore, HMGB1 might have a pivotal role in initiating and amplifying the neurogenic inflammatory cascade. We speculate that HMGB1 promotes neurogenic inflammation in the early stage of the infection and, successively, contributes to the pathogenesis of postinfection airway hyper-reactivity by modulating epithelialmesenchymal transition, and airway remodeling (37).

Critical evidence supporting the involvement of HMGB1 in the pathophysiology of RSV infection derives from the novel observation that selective inhibition of this molecule exerts a potent antiviral effect in human bronchial cells. Glycyrrhizin, a glycoside alkaloid extracted from Glycyrrhiza glabra roots, is made of the biologically active principle glycyrrhetic acid and two inactive components of glucuronic acid (38). By binding to the hydrophobic residues of HMGB1 box $\mathrm{A}$ and $\mathrm{B}$, glycyrrhizin inhibits the chemotactic and mitogenic function of HMGB1 and prevents HMGB1-DNA binding, thereby exerting anti-inflammatory and immunoregulatory effects in several non-infectious (39) and infectious (40) diseases. In particular, glycyrrhizin inhibits viral gene expression and replication, proinflammatory cells' recruitment, and T-lymphocyte responses (39).

In conclusion, our findings suggest that HMGB1 has a critical role in the initiation and maintenance of RSV replication in human bronchial epithelia and it is a promising target for monitoring and managing the infection in infants and children with bronchiolitis and pneumonia. The potential therapeutic effects of glycyrrhizin merit further exploration, especially in light of its safety profile, which lacks cytotoxicity even at high concentrations according to both experimental and clinical studies (41).

\section{ACKNOWLEDGMENTS}

We are particularly indebted to Mark Peeples (Nationwide Children's Hospital Research Institute, Columbus, $\mathrm{OH}$ ) and Peter Collins (National Institutes of Health, Bethesda, MD) for providing the RFP-expressing RSV.

\section{STATEMENT OF FINANCIAL SUPPORT}

This work was supported by NIH K08 Al112781 (F.R.) and NIH-NHLBI RO1HL061007 (G.P.). The funders had no role in study design, data collection and interpretation, or the decision to submit the work for publication.

Disclosure: The authors declare no conflict of interest.

\section{REFERENCES}

1. Wright M, Piedimonte G. Respiratory syncytial virus prevention and therapy: past, present, and future. Pediatr Pulmonol 2011;46:324-47.

2. Sigurs N, Bjarnason R, Sigurbergsson F, Kjellman B. Respiratory syncytial virus bronchiolitis in infancy is an important risk factor for asthma and allergy at age 7. Am J Respir Crit Care Med 2000;161:1501-7.
3. Piedimonte G, Walton C, Samsell L. Vertical transmission of respiratory syncytial virus modulates pre- and postnatal innervation and reactivity of rat airways. PLoS ONE 2013;8:e61309.

4. Hosakote YM, Brasier AR, Casola A, Garofalo RP, Kurosky A. Respiratory syncytial virus infection triggers epithelial HMGB1 release as a damage-associated molecular pattern promoting a monocytic inflammatory response. J Virol 2016;90:9618-31.

5. Hou CC, Zhao HJ, Cai SX, Li WJ, Tong WC, Liu LY. [Respiratory syncytial virus increases the expression and release of high mobility group Box-1 protein in the lung tissue of mice]. Nan Fang Yi Ke Da Xue Xue Bao 2010;30:700-3.

6. Harris HE, Raucci A. Alarmin(g) news about danger: workshop on innate danger signals and HMGB1. EMBO Rep 2006;7:774-8.

7. Campana L, Bosurgi L, Bianchi ME, Manfredi AA, Rovere-Querini P. Requirement of HMGB1 for stromal cell-derived factor-1/CXCL12dependent migration of macrophages and dendritic cells. J Leuk Biol 2009;86:609-15.

8. Javaherian K, Sadeghi M, Liu LF. Nonhistone proteins HMG1 and HMG2 unwind DNA double helix. Nucleic Acids Res 1979;6:3569-80.

9. Bustin M, Neihart NK. Antibodies against chromosomal HMG proteins stain the cytoplasm of mammalian cells. Cell 1979;16:181-9.

10. Gallucci S, Matzinger P. Danger signals: SOS to the immune system. Curr Opin Immunol 2001;13:114-9.

11. Yu M, Wang H, Ding A, et al. HMGB1 signals through toll-like receptor (TLR) 4 and TLR2. Shock 2006;26:174-9.

12. Ojo OO, Ryu MH, Jha A, Unruh H, Halayko AJ. High-mobility group box 1 promotes extracellular matrix synthesis and wound repair in human bronchial epithelial cells. Am J Physiol Lung Cell Mol Physiol 2015;309:L1354-66.

13. Kang R, Chen R, Zhang Q, et al. HMGB1 in health and disease. Mol Aspects Med 2014;40:1-116.

14. Schlegel TI CR, Garofalo RP, Palkowetz KH, et al. Release of high-mobility group box-1 (HMGB1) in the airways of children with viral lower respiratory tract infections. J Allergy Clin Immunol 2015;135: AB230.

15. Rezaee F, Meednu N, Emo JA, et al. Polyinosinic:polycytidylic acid induces protein kinase D-dependent disassembly of apical junctions and barrier dysfunction in airway epithelial cells. J Allergy Clin Immunol 2011;128:1216-24 e11.

16. Rezaee F, Desando SA, Ivanov AI, et al. Sustained protein kinase D activation mediates respiratory syncytial virus-induced airway barrier disruption. J Virol 2013;87:11088-95.

17. Hallak LK, Collins PL, Knudson W, Peeples ME. Iduronic acidcontaining glycosaminoglycans on target cells are required for efficient respiratory syncytial virus infection. Virology 2000;271:264-75.

18. Guerrero-Plata A, Casola A, Suarez G, et al. Differential response of dendritic cells to human metapneumovirus and respiratory syncytial virus. Am J Respir Cell Mol Biol 2006;34:320-9.

19. Techaarpornkul S, Barretto N, Peeples ME. Functional analysis of recombinant respiratory syncytial virus deletion mutants lacking the small hydrophobic and/or attachment glycoprotein gene. J Virol 2001;75:6825-34.

20. Piedimonte G. Pathophysiological mechanisms for the respiratory syncytial virus-reactive airway disease link. Respir Res 2002;3 (Suppl 1): S21-5.

21. Zeng R, Cui Y, Hai Y, Liu Y. Pattern recognition receptors for respiratory syncytial virus infection and design of vaccines. Virus Res 2012;167:138-45.

22. Chan JK, Roth J, Oppenheim JJ, et al. Alarmins: awaiting a clinical response. J Clin Invest 2012;122:2711-9.

23. Lotze MT, Zeh HJ, Rubartelli A, et al. The grateful dead: damageassociated molecular pattern molecules and reduction/oxidation regulate immunity. Immunol Rev 2007;220:60-81.

24. Wang $\mathrm{H}$, Bloom $\mathrm{O}$, Zhang $\mathrm{M}$, et al. HMG-1 as a late mediator of endotoxin lethality in mice. Science 1999;285:248-51.

25. Costello E, Saudan P, Winocour E, Pizer L, Beard P. High mobility group chromosomal protein 1 binds to the adeno-associated virus replication protein (Rep) and promotes Rep-mediated site-specific cleavage of DNA, ATPase activity and transcriptional repression. EMBO J 1997;16:5943-4. 


\section{Articles | Manti et al.}

26. Cotmore SF, Tattersall P. High-mobility group $1 / 2$ proteins are essential for initiating rolling-circle-type DNA replication at a parvovirus hairpin origin. J Virol 1998;72:8477-84.

27. van Zoelen MA, van der Sluijs KF, Achouiti A, et al. Receptor for advanced glycation end products is detrimental during influenza A virus pneumonia. Virology 2009;391:265-73.

28. Ito $\mathrm{Y}$, Torii $\mathrm{Y}$, Ohta $\mathrm{R}$, et al. Increased levels of cytokines and highmobility group box 1 are associated with the development of severe pneumonia, but not acute encephalopathy, in 2009 H1N1 influenzainfected children. Cytokine 2011;56:180-7.

29. Liang Y, Hou C, Kong J, et al. HMGB1 binding to receptor for advanced glycation end products enhances inflammatory responses of human bronchial epithelial cells by activating p38 MAPK and ERK1/2. Mol Cell Biochem 2015;405:63-71.

30. Arikkatt J, Ullah MA, Short KR, et al. RAGE deficiency predisposes mice to virus-induced paucigranulocytic asthma. Elife 2017;6; doi: 10.7554/ eLife.21199.

31. Castiglioni A, Canti V, Rovere-Querini P, Manfredi AA. High-mobility group box 1 (HMGB1) as a master regulator of innate immunity. Cell Tissue Res 2011;343:189-99.

32. van Zoelen MA, Yang H, Florquin S, et al. Role of toll-like receptors 2 and 4 , and the receptor for advanced glycation end products in high-mobility group box 1-induced inflammation in vivo. Shock 2009;31:280-4.

33. Seki E, Yoshizumi M, Tanaka R, et al. Cytokine profiles, signalling pathways and effects of fluticasone propionate in respiratory syncytial virus-infected human foetal lung fibroblasts. Cell Biol Int 2013;37:326-9.
34. Kalyan S, Chow AW. Linking innate and adaptive immunity: human Vgamma9Vdelta2 T cells enhance CD40 expression and HMGB-1 secretion. Mediators Inflamm 2009;2009:819408.

35. Lei C, Lin S, Zhang C, et al. Effects of high-mobility group box1 on cerebral angiogenesis and neurogenesis after intracerebral hemorrhage. Neuroscience 2013;229:12-9.

36. Othumpangat S, Gibson LF, Samsell L, Piedimonte G. NGF is an essential survival factor for bronchial epithelial cells during respiratory syncytial virus infection. PLoS ONE 2009;4:e6444.

37. Hou C, Kong J, Liang Y, et al. HMGB1 contributes to allergen-induced airway remodeling in a murine model of chronic asthma by modulating airway inflammation and activating lung fibroblasts. Cell Mol Immunol 2015;12:409-23.

38. Ploeger B, Mensinga T, Sips A, Seinen W, Meulenbelt J, DeJongh J. The pharmacokinetics of glycyrrhizic acid evaluated by physiologically based pharmacokinetic modeling. Drug Metab Rev 2001;33: 125-47.

39. Fiore C, Eisenhut M, Krausse R, et al. Antiviral effects of Glycyrrhiza species. Phytother Res 2008;22:141-8.

40. Matsumoto Y, Matsuura T, Aoyagi H, et al. Antiviral activity of glycyrrhizin against hepatitis C virus in vitro. PLoS ONE 2013;8:e68992.

41. van Rossum TG, Vulto AG, Hop WC, Brouwer JT, Niesters HG, Schalm SW. Intravenous glycyrrhizin for the treatment of chronic hepatitis $\mathrm{C}$ : a doubleblind, randomized, placebo-controlled phase I/II trial. J Gastroenterol Hepatol 1999;14:1093-9. 